ARTICLE

\title{
Recoverable plasticity in penta-twinned metallic nanowires governed by dislocation nucleation and retraction
}

Qingquan Qin ${ }^{1, \star}$, Sheng Yin ${ }^{2, \star}$, Guangming Cheng ${ }^{1}$, Xiaoyan $\mathrm{Li}^{3}$, Tzu-Hsuan Chang ${ }^{1}$, Gunther Richter ${ }^{4}$, Yong Zhu' \& Huajian Gao ${ }^{2}$

There has been relatively little study on time-dependent mechanical properties of nanowires, in spite of their importance for the design, fabrication and operation of nanoscale devices. Here we report a dislocation-mediated, time-dependent and fully reversible plastic behaviour in penta-twinned silver nanowires. In situ tensile experiments inside scanning and transmission electron microscopes show that penta-twinned silver nanowires undergo stress relaxation on loading and complete plastic strain recovery on unloading, while the same experiments on single-crystalline silver nanowires do not exhibit such a behaviour. Molecular dynamics simulations reveal that the observed behaviour in penta-twinned nanowires originates from the surface nucleation, propagation and retraction of partial dislocations. More specifically, vacancies reduce dislocation nucleation barrier, facilitating stress relaxation, while the twin boundaries and their intrinsic stress field promote retraction of partial dislocations, resulting in full strain recovery.

\footnotetext{
${ }^{1}$ Department of Mechanical and Aerospace Engineering, North Carolina State University, Raleigh, North Carolina 27695, USA. ${ }^{2}$ School of Engineering, Brown University, Providence, Rhode Island 02912, USA. ${ }^{3}$ Centre of Advanced Mechanics and Materials, Applied Mechanics Laboratory, Department of Engineering Mechanics, Tsinghua University, Beijing 100084, China. ${ }^{4}$ Max Planck Institute for Intelligent Systems, Heisenbergstrasse 3, D-70589 Stuttgart, Germany.

* These authors contributed equally to this work. Correspondence and requests for materials should be addressed to Y.Z. (email: yong_zhu@ncsu.edu) or to H.G. (email: huajian_gao@brown.edu).
} 
ne-dimensional (1D) nanostructures are widely regarded as among the most important building blocks for a broad range of applications including nanoelectronics, optoelectronics, energy harvesting and storage, ultrasensitive sensing and nanoelectromechanical devices ${ }^{1,2}$. 1D nanostructures commonly exhibit ultrahigh mechanical strength, which make them also ideal candidates for studying fundamental deformation mechanisms at the nanoscale ${ }^{3-5}$. In the case of metallic nanowires (NWs), dislocation nucleation from free surfaces has been identified as a dominant deformation mechanism, in contrast to the forest dislocation dynamics in bulk materials ${ }^{6-12}$. Recently, NWs with internal microstructures have received much interest. For instance, metallic NWs with different types of twin boundaries (TBs) have been studied, including parallel, inclined or perpendicular TBs with respect to the NW length direction ${ }^{13-18}$. However, there has been relatively little study on time-dependent responses of NWs under sustained or cyclic loadings, in spite of the obvious importance of this subject to the function and reliability of NW-based devices.

A number of recent experimental and computational studies have revealed substantial time-dependent and partially reversible deformation behaviours in small-scale materials with characteristic length scale below $100 \mathrm{~nm}^{19}$, especially nanocrystalline metal thin films $\mathrm{s}^{20-23}$. These behaviours have been attributed to the coupling and competition of reversible dislocation activities and grain boundary (GB)-mediated processes at different temperature and strain rates ${ }^{24-30}$. At relatively high temperatures and low strain rates, GB diffusion/sliding can dominate the timedependent behaviours, while dislocation nucleation and motion become more prevalent at lower temperatures and higher strain rates. More recently, atomistic simulations predicted a reversible transition between two crystal orientations during loading, leading to shape memory and pseudoelastic behaviours for several face-centred cubic single-crystalline metal NWs $\mathrm{N}^{7,31-33}$. This phenomenon was attributed to the formation of defect-free twins facilitated by relatively low stacking fault energy, nanometer-size scale and surface stress.

Here we report an unusual time-dependent deformation behaviour in penta-twinned Ag NWs, with stress relaxation on loading and complete strain recovery on unloading. Penta-twinned Ag NWs contain five TBs running in parallel to the NW length, exhibiting interesting mechanical properties such as strain hardening ${ }^{14,18}$. The critical role of the pentatwinned nanostructure is established by showing that the same phenomenon does not exist in single-crystalline $\mathrm{Ag}$ NWs. Large-scale atomistic simulations are then performed to explore the mechanisms underlying the observed behaviours in detail.

\section{Results}

Structural characterization of Ag NWs. Microstructure characterization of single-crystalline and penta-twinned Ag NWs is shown in Fig. 1. Both types of NWs are straight and uniform in diameter, with growth direction of $\langle 110\rangle$, as shown by transmission electron microscopy (TEM) images and selected area electron diffraction patterns in Fig. 1a,c. The single-crystalline Ag NWs exhibit a hexagonal cross-sectional morphology (inset of Fig. 1a). Figure $1 \mathrm{~b}$ shows a high-resolution TEM image of a single-crystalline Ag NW, indicating a perfect atomic structure along the longitudinal direction and a uniform atomic arrangement at $\{002\}$ surface facets. The penta-twinned Ag NWs contain a fivefold twinned nanostructure with five TBs running along $\{111\}$ planes in parallel to the longitudinal axis of the NWs and five surface facets along $\{100\}$ planes with a pentagonal crosssectional morphology (inset of Fig. 1c) ${ }^{18,34}$.

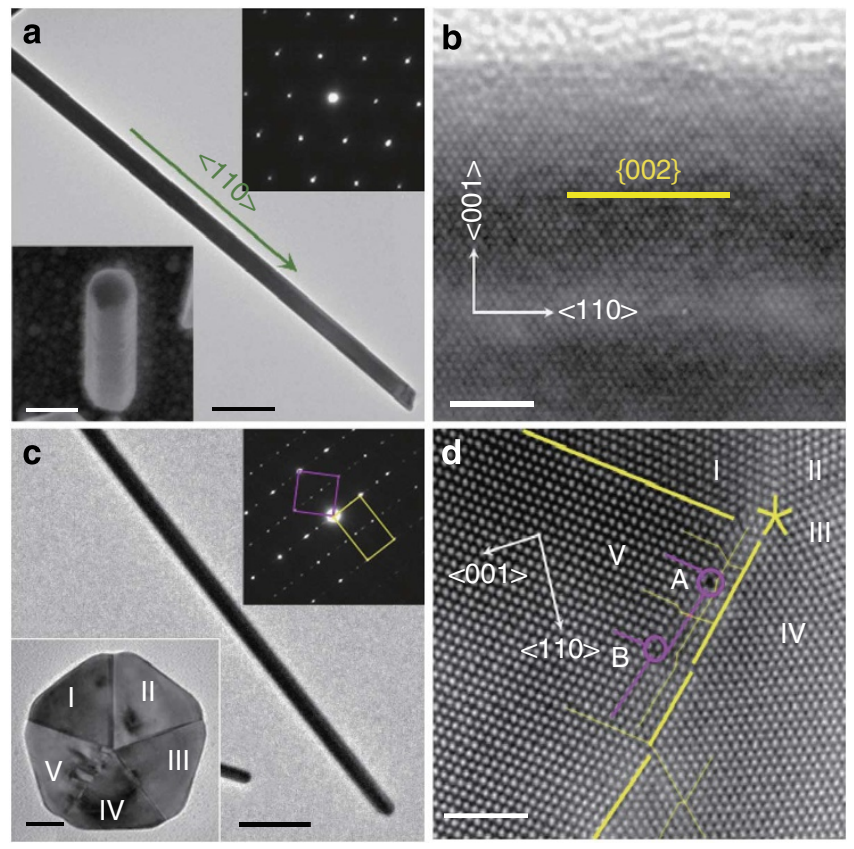

Figure 1 | Structural characterization of single-crystal and penta-twinned Ag NWs. (a,b) Low-magnification and high-resolution TEM images of single-crystal Ag NW with growth direction of $<110>$. Scale bar, 200 and $2 \mathrm{~nm}$, respectively. Right and left insets (scale bar, $100 \mathrm{~nm}$ ) in a show the selected area electron diffraction (SAED) pattern taken from $<110>$ zone axis and the hexagonal cross-sectional shape from SEM observation, respectively. (c) TEM image of $\mathrm{Ag}$ NWs showing fivefold twinned structure. Scale bar, $200 \mathrm{~nm}$. Right and left insets (scale bar, $20 \mathrm{~nm}$ ) in c display the corresponding SAED pattern and the pentagonal cross-sectional shape, respectively. Stacking faults along the boundary between grains IV and $V$ can be clearly seen in the left inset of c. (d) High-angle annular dark-field scanning TEM image of the cross-sectional sample showing the presence of vacancy defects near the boundary between grains IV and $\mathrm{V}$. The yellow star in $\mathbf{d}$ indicates the centre of the cross-sectional sample. Scale bar, $2 \mathrm{~nm}$.

The characteristics of the fivefold twins are illustrated from the cross-sectional view of the left inset of Fig. 1c,d. The five twin variants (TB-separated nanograins) are numbered from I to V. Stacking faults along the TB between twin variants IV and V can be clearly seen (see Supplementary Fig. 1). To further investigate the defect structures around the TBs, atomic-resolution highangle annular dark-field scanning TEM imaging is shown in Fig. 1d. In addition to the stacking faults, vacancy defects were identified near the TB, such as those marked by solid circles between twin variants IV and $\mathrm{V}$. The average density of the visible vacancy defects was estimated to be $\sim 2.23 \times 10^{25} \mathrm{~m}^{-3}(0.0375$ in percentage), which played an important role in our atomistic simulations. The formation of the vacancy defects is likely caused by interaction of partial dislocations during growth of the penta-twinned NWs. For instance, the vacancy defect marked by A can be formed by the interaction of two partials, $\frac{1}{3}\langle 111\rangle$ and $\frac{1}{6}\langle 11 \overline{2}\rangle$, based on the following reaction, $\frac{1}{3}\langle 111\rangle+\frac{1}{6}\langle 11 \overline{2}\rangle \rightarrow \frac{1}{3}\langle 11 \overline{1}\rangle+\frac{1}{6}\langle 112\rangle$. The $\frac{1}{3}\langle 11 \overline{1}\rangle$ partial is then locked, but the new $\frac{1}{6}\langle 112\rangle$ partial continues to move and interacts with another $\frac{1}{6}\langle 11 \overline{2}\rangle$ partial. As a result, a cascade of vacancy defects (for example, the one marked by B in Fig. 1d) can be formed.

In situ tensile testing of Ag NWs. We performed in situ tensile experiments inside scanning and transmission electron 
microscope (SEM/TEM) using a microelectromechanical system (MEMS)-based testing system that allows accurate measurement of both load and displacement simultaneously ${ }^{35-38}$, as shown in Fig. 2a. Load is applied using a thermal actuator on one side of the testing stage and measured using a differential capacitive sensor on the other side. Details on the load sensor calibration have been reported previously ${ }^{38}$. Displacement (and strain) is measured by digital image correlation of SEM images of two local markers on the specimen. This MEMS-based system has a strain resolution of $0.01 \%$ (gage length $2 \mu \mathrm{m}$ ) and a stress resolution of $1.4 \mathrm{MPa}$ (for example, for NW diameter of $104 \mathrm{~nm}$ ). First, stress relaxation experiments were conducted by loading the thermal

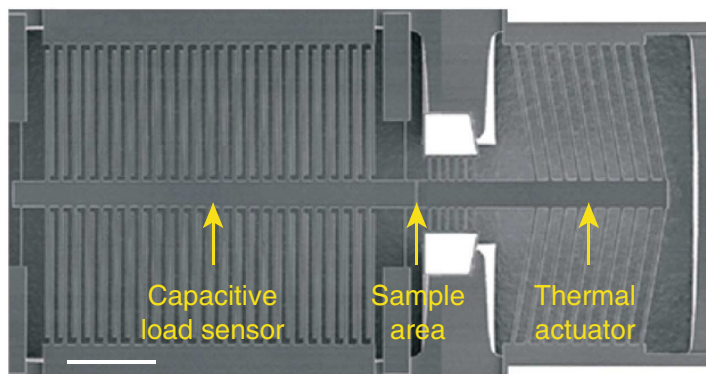

b

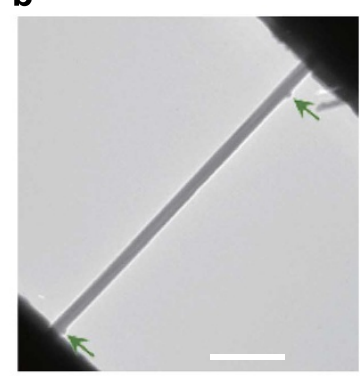

c
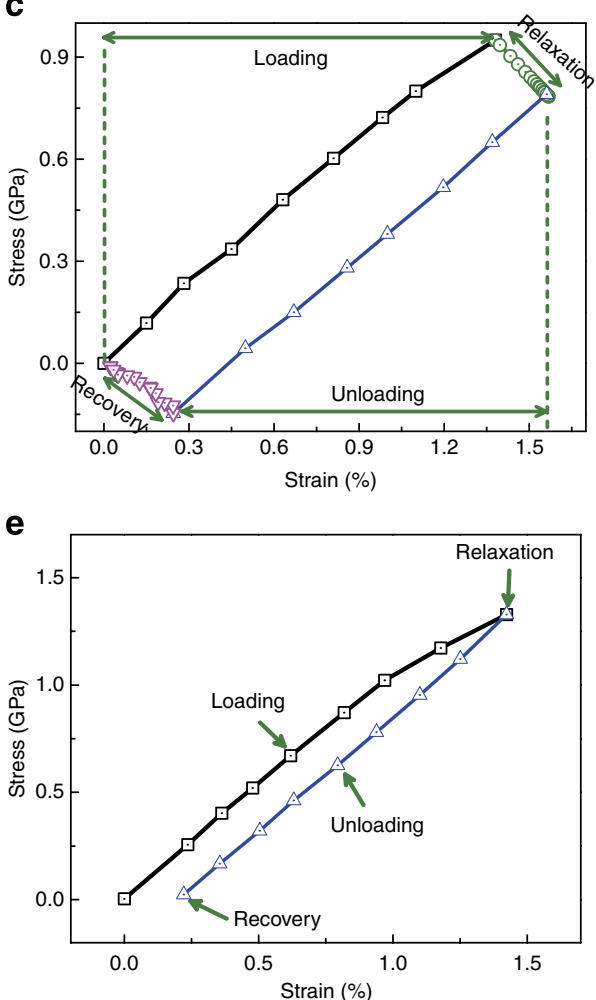

d

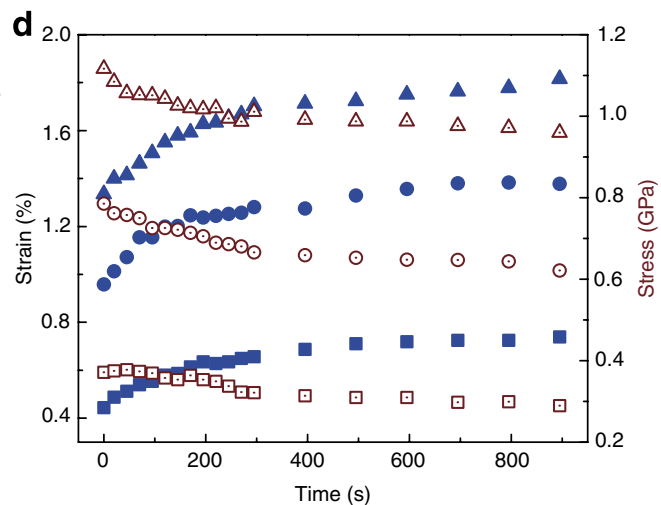

f

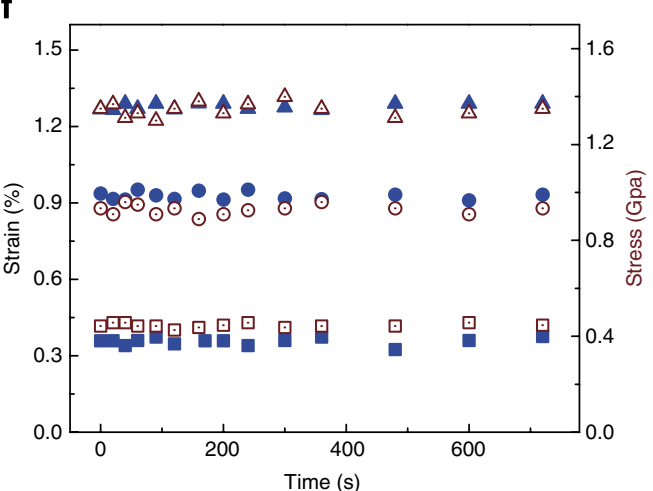

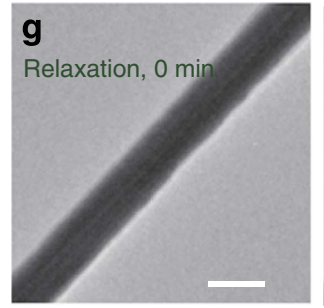
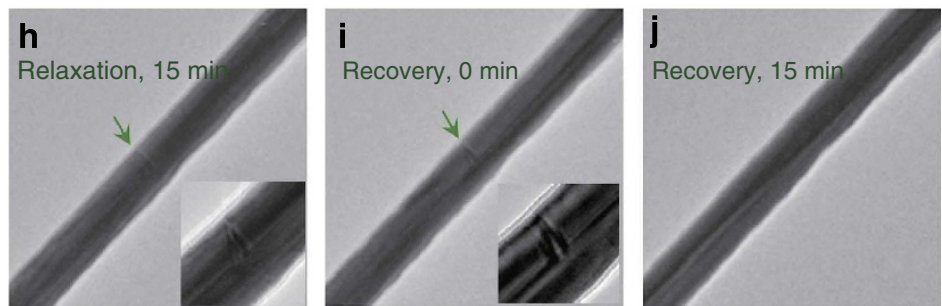

Figure 2 | In situ measurements of stress and strain evolutions in Ag NWs. (a) The MEMS stage used for in situ SEM and TEM tensile testing of Ag NWs. Scale bar, $200 \mathrm{~nm}$. (b) Low-magnification TEM image of a NW bridged between the actuator and the load sensor, with two marks (arrowed) for displacement measurement. Scale bar, $500 \mathrm{~nm}$. (c,e) Stress-strain curves for a penta-twinned Ag NW (120 nm in diameter) and a single-crystalline Ag NW ( $99 \mathrm{~nm}$ in diameter). Note that in both cases the relaxation and recovery steps took $15 \mathrm{~min}$ each. (d,f) Relaxation curves for a penta-twinned Ag NW (104 nm in diameter) and a single-crystalline $\mathrm{Ag} \mathrm{NW}$ ( $71 \mathrm{~nm}$ in diameter). Note: solid and open symbols correspond to the strain-time and stress-time relationships, respectively. Square, circle and triangle symbols correspond to first, second and third stress levels, respectively. (g-j) A series of TEM images extracted from Supplementary Movies 2 and 3 showing dislocation nucleation and annihilation in a penta-twinned Ag NW ( $85 \mathrm{~nm}$ in diameter). The arrows in $\mathbf{f}, \mathbf{g}$ point to the dislocation networks with high-magnification images shown in the insets. Scale bar, $150 \mathrm{~nm}$. 
actuator to a given displacement and while the actuator displacement was held constant, the specimen relaxed as a function of time. Since the experiment was not under true displacement control, the load on the specimen decreased and the specimen elongation increased at the same time. Subsequently, complete strain recovery was observed after unloading when turning off the thermal actuator. Note that the temperature rise at the specimen end due to the thermal actuator was less than $5^{\circ} \mathrm{C}$ for all the experiments reported here ${ }^{37}$.

Figure $2 \mathrm{c}$ shows the stress-strain response during a typical tensile test of a penta-twinned Ag NW in four steps: loading, relaxation, unloading and recovery (see also Supplementary Movie 1). During the loading step, the NW exhibited nearly linear response (slightly nonlinear when the applied stress is high). During the relaxation step, the stress decreased with time, while the strain increased. Figure $2 \mathrm{~d}$ shows the strain and stress as functions of time at three stress levels. When the initial stress is $\sim 0.37 \mathrm{GPa}$, the NW strain increased monotonically with time from 0.44 to $0.74 \%$, while the stress decreased monotonically from 371 to $290 \mathrm{MPa}$. On unloading, the strain in the NW completely recovers in about the same amount of time as the relaxation. The same procedure was repeated for the relaxation and recovery steps at two additional stress levels (with initial stresses of 0.79 and $1.12 \mathrm{GPa}$ ), corresponding to the circle and triangle symbols in Fig. 2d, respectively.

In situ TEM tensile testing indicated that the stress relaxation and strain recovery in a penta-twinned Ag NW are accompanied by nucleation and annihilation of dislocations, respectively, as shown in Fig. 2g-j. Before the relaxation step (Fig. 2g), there were no dislocations in the $\mathrm{NW}$ as the strain increased to $1.6 \%$. At the relaxation step, a dislocation network appeared in $<5$ min (see Supplementary Movie 2). The dislocations were terminated at the TBs (Fig. 2h). During the unloading step, the applied load gradually decreased to zero but the dislocations remained (Fig. 2i). In the recovery step, the dislocation network gradually retracted and suddenly disappeared after $\sim 8 \mathrm{~min}$ (Fig. 2j). After $15 \mathrm{~min}$, the NW was totally recovered (see Supplementary Movie 3). Overall, the same relaxation and recovery behaviours were observed in all five penta-twinned Ag NWs with different diameters $(48,85,104,120$ and $121 \mathrm{~nm})$ tested in either SEM or TEM (see Supplementary Fig. 2).

The same experiments were conducted for five single-crystalline Ag NWs with diameters of 71, 99, 111, 130 and $152 \mathrm{~nm}$ at similar stress levels for the penta-twinned Ag NWs, but neither stress relaxation (Fig. 2e,f) nor recovery of plastic strain (Fig. 2f) was observed in any of them (see Supplementary Movie 4).

MD simulations of stress relaxation and strain recovery. To reveal the underlying mechanisms behind the observed relaxation and recovery behaviours of penta-twinned Ag NWs, we performed a series of molecular dynamics (MD) simulations. Details of the simulation are supplied in Methods. It is known that stress relaxation is highly sensitive to the microstructures, particularly point defects $19,39,40$. Therefore, we introduced a population of vacancies with concentration from 0 to $2 \%$ in the simulated samples, based on experimental observations shown in Fig. 1d. To investigate stress relaxation behaviours, we first stretched the simulated samples to a strain of $1.8 \%$, and then monitored stress relaxation while the applied strain was held fixed. The simulation results showed no stress relaxation during a simulation time period over tens of nanoseconds in a vacancy-free NW. However, with $1 \%$ vacancies introduced in the penta-twinned NWs, the stress gradually decreased from an initial stress of $1.15 \mathrm{GPa}$ by about $200 \mathrm{MPa}$ (that is, $17.4 \%$ of the initial stress) in $2 \mathrm{~ns}$, as illustrated in Fig. 3a. The insets in Fig. 3a capture a sequence of
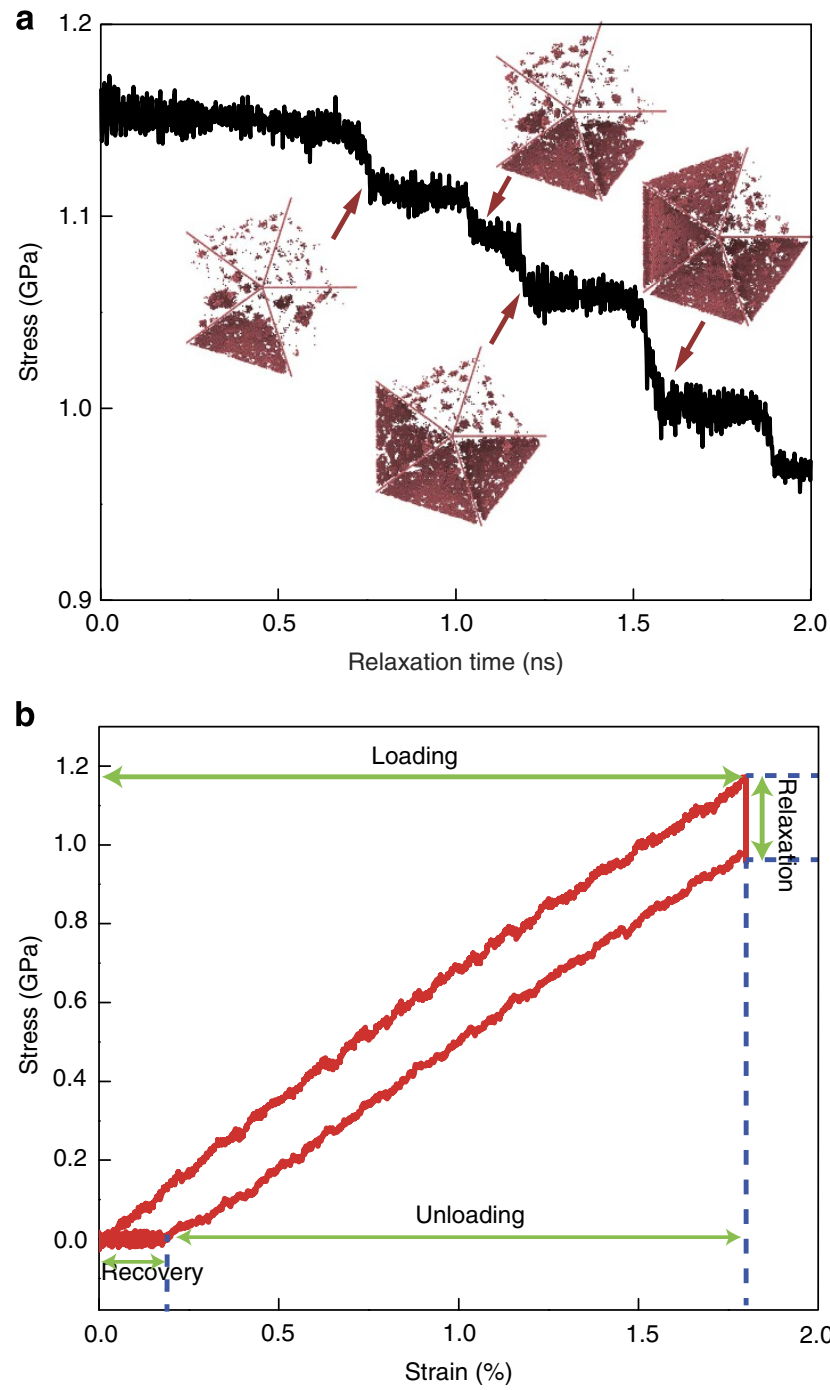

Figure 3 | Simulated stress relaxation and strain recovery in pentatwinned NWs. (a) Stress relaxation of a $30 \mathrm{~nm}$-diameter penta-twinned $\mathrm{Ag}$ NW. Each stress drop corresponds to a discrete event of dislocation nucleation shown in insets. (b) The stress-strain curve.

partial dislocation nucleation events in the TB-separated nanograins. The partial dislocation nucleation events exhibit a one-to-one correspondence with discrete stress drops in the stress relaxation profile shown in Fig. 3a, indicating that the stress relaxation is a direct consequence of dislocation nucleation in the penta-twinned nanostructure.

Similar to experiments, after stress relaxation the simulated samples were subsequently unloaded to a stress-free state. When the applied stress came down to zero, there was still a $0.21 \%$ of strain remaining in the sample, as shown in Fig. 3b. Continuing relaxation of the sample under zero stress resulted in complete recovery of the residue strain after $0.5 \mathrm{~ns}$ (see Fig. 4a). This phenomenon is very similar to the experimental observations. Figure $4 \mathrm{~b}, \mathrm{c}$ illustrates two snapshots of the deformed sample during relaxation and recovery, respectively. During stress relaxation, partial dislocation loops were found to nucleate spontaneously from aggregated vacancy clusters near free surface and then expand through the grain interiors separated by the five TBs. Each discrete dislocation nucleation event leads to a visible stress drop in our simulation. During stress relaxation, it was observed that several dislocation loops moved and leaned against 

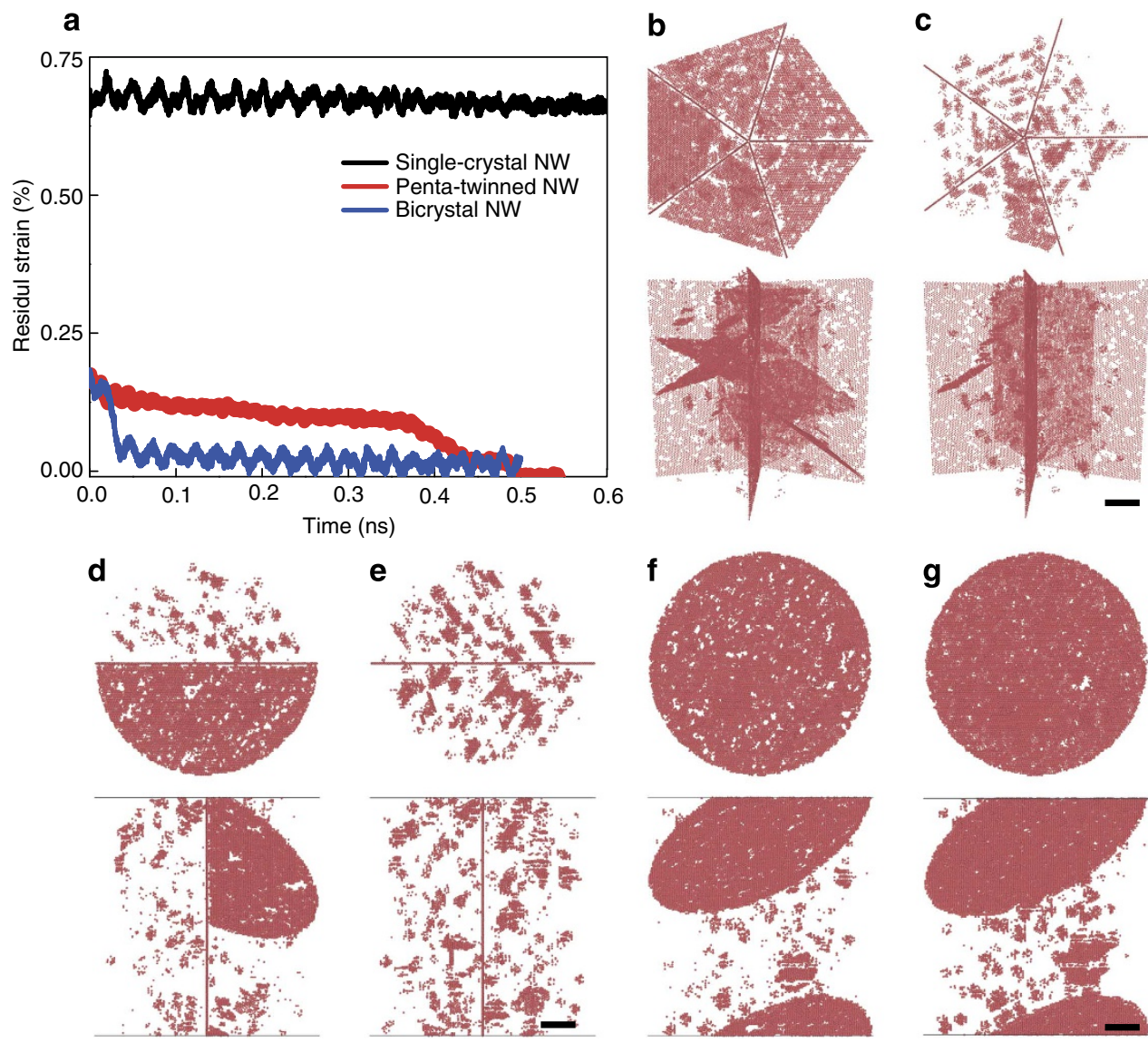

Figure 4 | Recoverable plasticity and associated dislocation activities. (a) Time-dependent strain recovery due to the reverse motion of partial dislocations. (b,c) Penta-twinned NW; (b) during relaxation, dislocations are nucleated and confined by the penta-twinned nanostructure; (c) dislocations retract and disappear during recovery. (d,e) Bi-crystalline NW; (d) during relaxation, a dislocation is impeded by the TB; (e) the dislocation retracts and disappears during recovery. $(\mathbf{f}, \mathbf{g})$ Single-crystalline NW; (f) during relaxation, dislocations travel through the sample interior and escapes out of the free surface, leaving behind a permanent stacking fault; (g) after unloading, the stacking fault still resides in the sample interior, resulting in permanent plastic deformation. Only hexagonal close-packed atoms are visible in (b-g) for clarity, upper parts are viewed from NW axis and lower parts are side view of NWs. Scale bar, $5 \mathrm{~nm}$.

TBs as the stress level went down and some dislocation segments were absorbed by, while others stayed close to, the TBs. During subsequent strain recovery after unloading, partial dislocation loops were seen to retract from the fivefold TBs under zero applied stress, resulting in complete strain recovery. Details of such a process are shown in Supplementary Movie 5. It is known that there exists a repulsive force between $\mathrm{TB}$ and curved dislocation loop ${ }^{41,42}$. When the external stress is removed, the repulsive force from the TBs appears to induce reverse motion of dislocations by pushing the non-inserted segments as well as extracting the inserted segments from the TBs back towards where the dislocations were nucleated. Moreover, we have analysed the Peach-Koehler force exerted on the partial dislocations by the inhomogeneous intrinsic stress field of the fivefold twin and found that it will also act as a driving force for dislocation retraction during strain recovery (see Supplementary Note 1; Supplementary Figs 3-5). In addition, the intrinsic stress field of the fivefold twin has a certain gradient from the core to the free surface, which may assist vacancy diffusion.

While it is difficult to fully reveal the initial point defects in the NW samples, careful TEM examinations identified their presence near TBs (Fig. 1d). In accordance with this observation, we performed additional simulations with initial defects created in two neighbouring twin variants in the penta-twinned NWs. After equilibration, the overall vacancy concentration is measured to be around $0.1 \%$. In such simulated samples with localized vacancy distribution near a $\mathrm{TB}$, we also observed stress relaxation and plastic strain recovery (see Supplementary Movie 6).

\section{Discussion}

In our simulations, the stress relaxation is significantly affected by vacancies in the NWs. When the vacancy concentration is increased by $0.5 \%$, stress relaxation becomes more pronounced. Under a given initial stress, higher vacancy concentration leads to more dislocation nucleation events and larger stress drops (see Supplementary Fig. 6). To further understand how vacancy facilitates dislocation nucleation, we computed the generalized planar fault curves (essentially reflecting plastic deformation map) of Ag under different vacancy concentrations (see Supplementary Fig. 7). The results indicate that the vacancy reduces the energy barrier associated with the nucleation of leading partial dislocations in Ag. The relevant details are given in Supplementary Note 2. Remarkably, the ratio of unstable stacking fault energy to stacking fault energy $\gamma_{\mathrm{sf}} / \gamma_{\text {usf }}$ decreases as vacancy concentration rises (see Supplementary Fig. 7). On the basis of previous studies 43,44 , such a phenomenon implies that full dislocations are getting harder to nucleate and the slip of energetically more favourable leading partials becomes a 
controlling plastic deformation mechanism. In addition, we performed a simulation for the uniaxial tension of a simple Ag block with (001) free surfaces to examine the influence of vacancy concentration on dislocation nucleation (see Supplementary Fig. 8). It is found that the first partial dislocation nucleates from the free surface at $4.15 \%$ strain in a perfect block, while this critical strain drops to $2.50 \%$ at vacancy concentration of $1 \%$. These results indicate that the presence and aggregation of vacancies can promote the nucleation as well as operation of leading partial dislocations as a dominating deformation mechanism.

Previous studies ${ }^{25-29}$ showed that plastic strain recovery in thin films can be attributed to dislocation- and GB-mediated processes. In penta-twinned NWs, there are no regular GBs and the TBs have low energy, high symmetry, coherent atomic structure and one side geometrically constrained in the centre of the NWs. In this nanostructure, the sliding and diffusion processes should be suppressed to a large extent. Therefore, the controlling mechanism for the observed strain recovery behaviour can only be attributed to TB-dislocation interactions. The fivefold TBs separate the NW into five nanograins, which confine the length of dislocation segments as well as the mean free path of vacancy diffusion. More importantly, TBs are effective obstacles for dislocations and produce a repulsive force to drive the reverse motion of dislocations during unloading. In addition to these effects, the inhomogeneous stress field generated by the fivefold twin may also benefit vacancy diffusion and reversible dislocation motion.

To further demonstrate the unique role of TBs in the recovery behaviour of NWs, we performed two additional simulations for a single-crystalline NW and a bi-crystalline NW with a single TB in the middle-a mono-twinned NW. To facilitate dislocation nucleation in simulation, these two samples have the same vacancy concentration of $1.0 \%$. Repeating the simulations as before but relaxing the two NWs from an initial strain of $2.0 \%$, we observed nearly full plastic strain recovery in the mono-twinned NW, but not in the single-crystalline NW, as illustrated in Fig. 4a. In the mono-twinned NW, the TB effectively blocks the motion of leading partials, preventing them from escaping out of the sample (see Fig. 4d,e) and allowing them to retract back from the TB under the residual internal stress field on unloading, leading to plastic strain recovery, albeit not as complete as the pentatwinned case. In contrast, in the single-crystalline NW, dislocations nucleated from the free surface, travelled across the NW and eventually escaped out of the sample, leaving a permanent surface step (see Supplementary Movies 7 and 8). In this case, the dislocation motion, and the associated deformation, becomes irreversible during subsequent unloading. Besides the twinned $\mathrm{NW}$, we have also investigated bi-crystalline NWs with different types of regular GBs (such as low-angle/high-angle tilt GBs and mixed GB). It is found that the tilt and mixed GBs either failed to block the dislocation or trapped the dislocation, resulting in no strain recovery (see Supplementary Note 3; Supplementary Fig. 9; Supplementary Movies 9 and 10). These studies further demonstrate that the TBs play an essential role in the reverse motion of partial dislocations, which lead to the observed plastic strain recovery in the penta-twinned Ag NWs. It is noted that, in penta-twinned NWs, fivefold TBs divide the $\mathrm{NW}$ interior into five nanograins, which can prevent the escape of dislocations from free surface and facilitate dislocation retraction more effectively compared with a single $\mathrm{TB}$ in bi-crystalline NWs. In addition, the intrinsic residual stress field induced by the fivefold twin also favours dislocation retraction on unloading.

We have also investigated the influence of sample diameter on the observed deformation behaviour in penta-twinned Ag NWs (see Supplementary Figs 10 and 11; Supplementary Note 4), with results showing that the characteristic time scale (that is, relaxation time) for the observed behaviour depends on the sample diameter (see Supplementary Tables 1 and 2). As the sample diameter decreases, the relaxation time increases, in agreement with both our experiments and simulations. Such size dependence on the relaxation time is essentially attributed to the size effect of dislocation nucleation. The relaxation time should depend on the activation energy and stress for dislocation nucleation. Under the same temperature and strain rate, the critical stress for dislocation nucleation from free surfaces (in the case of NWs) was found to be inversely proportional to the NW diameter. Therefore, stress relaxation should be less prominent in smaller samples than in larger samples. A more detailed study on the size dependence of the relaxation and recovery behaviours will be performed in the future.

Plastic deformation in metals generally consists of two components: an athermal part and a thermal part. The former, driven by the long-range internal stress, has a weak temperature dependence, while the latter accounts for thermally activated processes that are usually sensitive to both temperature and strain rate. In our experiments and simulations reported above, the NWs did not reach the athermal yield point during loading. It was during the relaxation step that the thermally activated nucleation of partial dislocations was observed.

To investigate whether the phenomenon of recoverable plasticity also occurs for similar samples beyond the athermal plastic yield point, we have performed additional MD simulations to investigate the loading-unloading behaviour of the pentatwinned Ag NWs under much larger strains. The NWs were loaded beyond the athermal yielding point such that partial dislocations are nucleated even without the relaxation step. In the first simulation, the sample was loaded to $4.5 \%$ strain, which is only slightly beyond the athermal yielding point (see Supplementary Fig. 12). In this case, a number of partial dislocations were nucleated during loading and then retracted back to their original nucleation sites during unloading, still resulting in full plastic strain recovery. During this process, none of the partial dislocations cut across the TBs. In the second simulation, the sample was loaded to about $10 \%$ strain, corresponding to very large plastic deformation before unloading (see Supplementary Fig. 13). There was only about $1 \%$ strain recovered during unloading. In this case, the applied stress was large enough to allow dislocations to cut across the TBs, and multiple slip systems were activated, leading to reactions and entanglement between dislocations from different slip systems. As a result, more complex and disordered dislocation structures prevented full strain recovery and only $1 \%$ strain was observed to recover, reminiscent of the classical Bauschinger's effect.

In summary, we have discovered an unusual dislocation-based stress relaxation and strain recovery behaviour in penta-twinned Ag NWs via in situ SEM/TEM tensile testing, TEM microstructural characterizations and large-scale MD simulations. This behaviour consists of two facets: stress relaxation on loading and complete plastic strain recovery on unloading. Our studies indicate that stress relaxation originates from the nucleation of leading partial dislocations assisted by vacancy diffusion, while the complete strain recovery is due to the reverse motion of partial dislocations driven by the repulsive force from the TBs and the intrinsic stress field due to the fivefold twin. The simulations demonstrate that the TBs play a crucial role in the observed phenomenon in penta-twinned NWs, as bi-crystalline NWs with a single TB exhibit a similar behaviour, while single-crystalline NWs do not. It is noted that diffusion and sliding processes associated with GBs should be suppressed in 
penta-twinned NWs. Therefore, in contrast to previous studies in nanocrystalline metals due to a cooperation of dislocation- and GB-mediated processes, the present study demonstrates a new type of stress relaxation and full plastic strain recovery induced by TB-dislocation interactions. Our study opens up a promising prospect of designing 1D nanostructures with time-dependent strain recovery capabilities.

\section{Methods}

Sample synthesis and characterization. Penta-twinned Ag NWs were synthesized by reducing $\mathrm{AgNO}_{3}$ with ethylene glycol in the presence of polyvinyl pyrrolidone. More details of the NW synthesis process are provided elsewhere ${ }^{45}$. The solution of Ag NWs was diluted with deionized water and then purified by centrifugation. Single-crystalline Ag NWs were synthesized by physical vapour deposition inside a molecular beam epitaxy system under ultrahigh vacuum condition and substrate temperature of $700^{\circ} \mathrm{C}$. More details of the NW synthesis process are provided elsewhere ${ }^{4}$

Conventional TEM observations were performed on JEOL 2010F operated at $200 \mathrm{kV}$. Atomic-resolution high-angle annular dark-field scanning TEM imaging was performed on a probe-corrected FEI Titan $\mathrm{G}^{2} 60-300 \mathrm{kV}$ S/TEM equipped with an extreme field emission gun (X-FEG) electron source operated at $200 \mathrm{kV}$.

Cross-section TEM samples were prepared by embedding Ag NWs into Gatan G1 epoxy with a $\phi 3 \mathrm{Cu}$ tube, cutting the specimen discs with a thickness of $\sim 0.5 \mathrm{~mm}$, mechanically polishing with Allied Multiprep System and finally ion milling the sample via Gatan 791 PIPS while cooling with liquid nitrogen.

In situ SEM/TEM mechanical testing. In situ mechanical testing inside a SEM or TEM was carried out using a MEMS-based material-testing system, which consists of a thermal actuator, a capacitive load sensor and a gap in between for mounting samples. The displacement markers are deposited using electron beam-induced deposition of carbon. Ag NWs were mounted on the testing stage using a nanomanipulator (Klocke Nanotechnik, Germany) inside a FEI Nova 600 dual beam and clamped by carbon deposition. The loading and unloading strain rates for in situ SEM tensile testing were $\sim 0.1 \% \mathrm{~s}^{-1}$. During loading, the actuator was loaded incrementally to a prescribed displacement. During relaxation, the actuator displacement was held constant, and the specimen relaxed as a function of time. More specifically, the load on the specimen decreased and the specimen elongation increased at the same time. During recovery, the actuator was turned off and retracted to the original position.

In situ TEM mechanical testing was performed on JEOL 2010F operated at $200 \mathrm{kV}$. The loading and unloading strain rates were $\sim 0.005 \% \mathrm{~s}^{-1}$. Lowmagnification images were recorded at a fixed condense current to minimize the focus change. Real-time videos were recorded at a speed of three frames per second.

Atomistic simulations. Large-scale MD simulations were performed using the software package LAMMPS ${ }^{46}$. Three kinds of NW samples are generated: a pentatwinned NW with a fivefold twin, a bi-crystalline NW with a single coherent TB in the middle and a single-crystalline NW. Supplementary Figure 14 shows the atomic configurations of the three kinds of simulated samples. Each sample is $30 \mathrm{~nm}$ in diameter and $30 \mathrm{~nm}$ in length and contains about 1.3 million atoms. The embedded atom method potential for $\mathrm{Ag}^{47}$ is used to describe the interatomic interactions. The vacancies are introduced by randomly removing atoms out of the samples.

The samples are initially relaxed and equilibrated at an elevated temperature of $800 \mathrm{~K}$ (about $65 \%$ of the melting point of $\mathrm{Ag}$ ) for $80 \mathrm{ps}$ using the Nosé-Hoover thermostat and barostat ${ }^{48}$. Periodic boundary condition is imposed along the axial direction (that is, the loading direction $<110>$ ) of all simulated samples. The samples are first stretched to a certain strain of 1.8 or $2 \%$ at a constant strain rate of $10^{8} \mathrm{~s}^{-1}$ under NVT ensemble (canonical ensemble), and then relaxed for several nanoseconds under NVT ensemble while holding the strain fixed. In the relaxation process, we monitor the variation of the axial stress by averaging the virial stresses ${ }^{49}$ over all atoms in the samples. Note that we have used an elevated temperature of $800 \mathrm{~K}$ in our MD simulations to accelerate the thermally activated processes of vacancy diffusion and the associated dislocation nucleation within the time scale of MD simulations. To examine the reversibility of deformation (related to the reverse motion of dislocations) and measure the residual strain, we unload the elongated samples at a strain rate of $-10^{8} \mathrm{~s}^{-1}$ until the axial stress in the simulated samples approaches zero. Subsequently, the samples are further relaxed at the equilibrium state while the axial stress is kept zero by a Nosé-Hoover barostat. The residual strain is calculated by comparing the initial and final lengths of the samples. Throughout the simulations, the temperature is kept constant at $800 \mathrm{~K}$ by the Nosé-Hoover thermostat. The loading and unloading processes are designed to mimic the real experimental testing apart from much higher strain rates.

To identify defects during deformation of the samples, we paint atoms with different crystalline order in different colours using a common neighbour analysis. The green-coloured atoms stand for atoms with face-centred cubic symmetry, the red ones those with hexagonal close-packed symmetry and the grey ones those at dislocation cores, surfaces and point defects.

\section{References}

1. Lieber, C. M. \& Wang, Z. L. Functional nanowires. MRS Bull. 32, 99-108 (2007).

2. Xia, Y. et al. One-dimensional nanostructures: synthesis, characterization, and applications. Adv. Mater. 15, 353-389 (2003).

3. Greer, J. R. \& De Hosson, J. T. M. Plasticity in small-sized metallic systems: intrinsic versus extrinsic size effect. Prog. Mater. Sci. 56, 654-724 (2011).

4. Richter, G. et al. Ultrahigh strength single crystalline nanowhiskers grown by physical vapor deposition. Nano Lett. 9, 3048-3052 (2009).

5. Zhu, T. \& Li, J. Ultra-strength materials. Prog. Mater. Sci. 55, 710-757 (2010).

6. Lu, Y., Huang, J. Y., Wang, C., Sun, S. \& Lou, J. Cold welding of ultrathin gold nanowires. Nat. Nanotechnol. 5, 218-224 (2010).

7. Park, H. S., Gall, K. \& Zimmerman, J. A. Shape memory and pseudoelasticity in metal nanowires. Phys. Rev. Lett. 95, 255504 (2005).

8. Park, H. S., Gall, K. \& Zimmerman, J. A. Deformation of FCC nanowires by twinning and slip. J. Mech. Phys. Solids 54, 1862-1881 (2006).

9. Weinberger, C. R. \& Cai, W. Plasticity of metal nanowires. J. Mater. Chem. 22, 3277-3292 (2012).

10. Yue, Y. et al. Quantitative evidence of crossover toward partial dislocation mediated plasticity in copper single crystalline nanowires. Nano Lett. 12, 4045-4049 (2012).

11. Zheng, H. et al. Discrete plasticity in sub-10-nm-sized gold crystals. Nat. Commun. 1, 144 (2010)

12. Zhu, T., Li, J., Samanta, A., Leach, A. \& Gall, K. Temperature and strain-rate dependence of surface dislocation nucleation. Phys. Rev. Lett. 100, 025502 (2008).

13. Deng, C. \& Sansoz, F. Fundamental differences in the plasticity of periodically twinned nanowires in $\mathrm{Au}, \mathrm{Ag}, \mathrm{Al}, \mathrm{Cu}, \mathrm{Pb}$ and $\mathrm{Ni}$. Acta Mater. 57, 6090-6101 (2009).

14. Filleter, T. et al. Nucleation-controlled distributed plasticity in penta-twinned silver nanowires. Small 8, 2986-2993 (2012).

15. Jang, D., Li, X., Gao, H. \& Greer, J. R. Deformation mechanisms in nanotwinned metal nanopillars. Nat. Nanotechnol. 7, 594-601 (2012).

16. Lu, Y., Song, J., Huang, J. Y. \& Lou, J. Fracture of sub-20 nm ultrathin gold nanowires. Adv. Funct. Mater. 21, 3982-3989 (2011).

17. Wang, J. et al. Near-ideal theoretical strength in gold nanowires containing angstrom scale twins. Nat. Commun. 4, 1742 (2013).

18. Zhu, Y. et al. Size effects on elasticity, yielding, and fracture of silver nanowires: in situ experiments. Phys. Rev. B 85, 045443 (2012).

19. Baker, S. P., Vinci, R. P. \& Arias, T. Elastic and anelastic behavior of materials in small dimensions. MRS Bull. 27, 26-29 (2002).

20. Choi, D.-h., Kim, H. \& Nix, W. D. Anelasticity and damping of thin aluminum films on silicon substrates. J. Microelectromech. Syst. 13, 230-237 (2004).

21. Haque, M. \& Saif, M. Thermo-mechanical properties of nano-scale freestanding aluminum films. Thin Solid Films 484, 364-368 (2005).

22. Karanjgaokar, N., Oh, C.-S., Lambros, J. \& Chasiotis, I. Inelastic deformation of nanocrystalline $\mathrm{Au}$ thin films as a function of temperature and strain rate. Acta Mater. 60, 5352-5361 (2012).

23. Lang, M. et al. Measurement of elastic and anelastic properties of nanocrystalline metals. Nanostruct. Mater. 12, 811-816 (1999).

24. Chen, B. et al. Anelastic behavior in GaAs semiconductor nanowires. Nano Lett. 13, 3169-3172 (2013).

25. Li, X., Wei, Y., Yang, W. \& Gao, H. Competing grain-boundary-and dislocation-mediated mechanisms in plastic strain recovery in nanocrystalline aluminum. Proc. Natl Acad. Sci. USA 106, 16108-16113 (2009).

26. Rajagopalan, J., Han, J. H. \& Saif, M. T. A. Plastic deformation recovery in freestanding nanocrystalline aluminum and gold thin films. Science 315, 1831-1834 (2007).

27. Wei, X. \& Kysar, J. W. Residual plastic strain recovery driven grain boundary diffusion in nanocrystalline thin films. Acta Mater. 59, 3937-3945 (2011).

28. Wei, Y., Bower, A. F. \& Gao, H. Recoverable creep deformation due to heterogeneous grain-boundary diffusion and sliding. Scr. Mater. 57, 933-936 (2007)

29. Wei, Y., Bower, A. F. \& Gao, H. Recoverable creep deformation and transient local stress concentration due to heterogeneous grain-boundary diffusion and sliding in polycrystalline solids. J. Mech. Phys. Solids 56, 1460-1483 (2008).

30. Yamakov, V., Wolf, D., Phillpot, S. R., Mukherjee, A. K. \& Gleiter, H. Dislocation processes in the deformation of nanocrystalline aluminium by molecular-dynamics simulation. Nat. Mater. 1, 45-49 (2002).

31. Diao, J., Gall, K. \& Dunn, M. L. Surface-stress-induced phase transformation in metal nanowires. Nat. Mater. 2, 656-660 (2003).

32. Liang, W., Srolovitz, D. J. \& Zhou, M. A micromechanical continuum model for the tensile behavior of shape memory metal nanowires. J. Mech. Phys. Solids 55, 1729-1761 (2007).

33. Liang, W. \& Zhou, M. Atomistic simulations reveal shape memory of fcc metal nanowires. Phys. Rev. B 73, 115409 (2006).

34. Chen, H. et al. Transmission-electron-microscopy study on fivefold twinned silver nanorods. J. Phys. Chem. B 108, 12038-12043 (2004). 
35. Qin, Q. \& Zhu, Y. Temperature control in thermal microactuators with applications to in-situ nanomechanical testing. Appl. Phys. Lett. 102, 013101-013105 (2013).

36. Zhu, Y., Corigliano, A. \& Espinosa, H. D. A thermal actuator for nanoscale in situ microscopy testing: design and characterization. J. Micromech. Microeng. 16, 242-253 (2006)

37. Zhu, Y. \& Espinosa, H. D. An electromechanical material testing system for in situ electron microscopy and applications. Proc. Natl Acad. Sci. USA 102, 14503-14508 (2005)

38. Zhu, Y., Moldovan, N. \& Espinosa, H. D. A microelectromechanical load sensor for in situ electron and x-ray microscopy tensile testing of nanostructures. Appl. Phys. Lett. 86, 013506 (2005).

39. Nowick, A. S. Anelastic Relaxation in Crystalline Solids. Vol. 1 (Elsevier, 1972).

40. Zener, C. Elasticity and Anelasticity of Metals (Univ. of Chicago Press, 1948).

41. Chen, Z., Jin, Z. \& Gao, H. Repulsive force between screw dislocation and coherent twin boundary in aluminum and copper. Phys. Rev. B 75, 212104 (2007).

42. Deng, C. \& Sansoz, F. Repulsive force of twin boundary on curved dislocations and its role on the yielding of twinned nanowires. Scr. Mater. 63, 50-53 (2010).

43. Frøseth, A., Derlet, P. \& Van Swygenhoven, H. Dislocations emitted from nanocrystalline grain boundaries: nucleation and splitting distance. Acta Mater. 52, 5863-5870 (2004).

44. Van Swygenhoven, H., Derlet, P. \& Frøseth, A. Stacking fault energies and slip in nanocrystalline metals. Nat. Mater. 3, 399-403 (2004).

45. Wiley, B., Sun, Y. \& Xia, Y. Synthesis of silver nanostructures with controlled shapes and properties. Acc. Chem. Res. 40, 1067-1076 (2007).

46. Plimpton, S. Fast parallel algorithms for short-range molecular-dynamics. J. Comput. Phys. 117, 1-19 (1995).

47. Williams, P., Mishin, Y. \& Hamilton, J. An embedded-atom potential for the Cu-Ag system. Model. Simul. Mater. Sci. Eng. 14, 817-833 (2006).

48. Nose, S. A unified formulation of the constant temperature moleculardynamics methods. J. Chem. Phys. 81, 511-519 (1984).

49. Tsai, D. The virial theorem and stress calculation in molecular dynamics. J. Chem. Phys. 70, 1375-1382 (1979).

\section{Acknowledgements}

Q.Q., G.C., T.-H.C. and Y.Z. acknowledge support by the National Science Foundation (NSF) under Awards CMMI-1030637 and 1301193, and the use of the Analytical Instrumentation Facility at North Carolina State University, which is supported by the State of North Carolina and NSF. S.Y., X.L. and H.G. acknowledge support by NSF through Award CMMI-1161749 and computational support by the National Institute for Computational Sciences (NICS) through Grant MS090046. X.L. also acknowledges support by the Chinese 1000-talent plan and the NSFC grant 11372152 .

\section{Author contributions}

Y.Z. and H.G. conceived the project and designed the experiments and simulations; Q.Q., G.C. and T.-H.C. performed the in situ experiments; S.Y. and X.L. performed the atomistic simulations; G.R. provided the single-crystalline Ag NW samples; all authors analysed the data, discussed the results and wrote the manuscript.

\section{Additional information}

Supplementary Information accompanies this paper at http://www.nature.com/ naturecommunications

Competing financial interests: The authors declare no competing financial interests.

Reprints and permission information is available online at http://npg.nature.com/ reprintsandpermissions/

How to cite this article: Qin, Q. et al. Recoverable plasticity in penta-twinned metallic nanowires governed by dislocation nucleation and retraction. Nat. Commun. 6:5983 doi: 10.1038/ncomms6983 (2015).

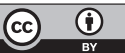

This work is licensed under a Creative Commons Attribution 4.0 International License. The images or other third party material in this article are included in the article's Creative Commons license, unless indicated otherwise in the credit line; if the material is not included under the Creative Commons license, users will need to obtain permission from the license holder to reproduce the material. To view a copy of this license, visit http://creativecommons.org/licenses/by/4.0/ 